\title{
Heart Failure with Preserved Ejection Fraction
}

\author{
Ahmed Tageldien Abdellah, MD, FRSM \\ Cardiology Department, Faculty of Medicine, Suez Canal University, Egypt
}

\begin{abstract}
Heart failure (HF) is a worldwide growing epidemic, which affects $2-3 \%$ of the adults. Notably, HF manifestations can exist in patients with preserved left ventricular ejection fraction (HFPEF) which constitutes up to $50 \%$ of all HF patients. HFPEF is a heterogeneous entity with a number of key pathophysiologic mechanisms including impaired diastolic relaxation, abnormal filling, myocardial restriction and stiffness, longitudinal axis ventricular dysfunction, or right ventricular dysfunction. It is characterized by impaired peak oxygen consumption, elevated $\mathrm{N}$ terminal pro $\mathrm{B}$ type natriuretic peptide (NT pro-BNP) which represents the main physiologic and neurohormonal changes. HFPEF is more common in older age and in women, especially in patients with history of hypertension, atrial fibrillation (AF), coronary artery disease (CAD) and diabetes. Yet, it is mainly a diagnosis of exclusion, with heterogeneous differential diagnosis, high rate of hospitalization, and comparable mortality to HF with systolic dysfunction (SHF).
\end{abstract}

Keywords: heart failure-preserved ejection fraction - diastolic function -pathophysiology - diagnosis.

\section{Introduction}

Heart failure (HF) represents a major and growing public health problem, affecting $2 \%-3 \%$ of adults in developed countries ${ }^{(1)}$. HF is no longer synonymous to diminished contractility of the LV or reduced LVEF, this concept was postulated by Kessler in 1988 in patients presented with pulmonary congestion with preserved LVEF (HFPEF) and evidence of significant diastolic dysfunction $^{(2)}$. Afterwards, a significant body of evidence has emerged showing that classical signs and symptoms of HF may present despite a normal LV size and EF. The prevalence of HFPEF is estimated to constitute about $50 \%$ of patients who have a clinical diagnosis of HF. HFPEF represents a heterogeneous group of HF population, which is only similar in preservation of LVEF, otherwise they have variable characteristics.
Diastolic HF, or HF with normal ejection fraction have been synonymous to HFPEF, but the latter is the most commonly used term for this syndrome ${ }^{(3)}$.

Similar to the physiologic and neurohormonal changes that exist in SHF patients, HFPEF patients are characterized by impaired peak oxygen consumption, and elevated circulating neurohormones, including B-type natriuretic peptide (BNP) and norepinephrine. Principal pathophysiologic abnormalities include abnormal renal sodium handling, fluid retention, and reduced vascular compliance, increased myocardial and arterial stiffness ${ }^{(4)}$. These changes lead to myocardial restriction, impaired diastolic relaxation, abnormal filling, longitudinal axis ventricular dysfunction, or right ventricular dysfunction ${ }^{(5)}$. These changes might coexist with variable degree 
over time, ranging from unremarkable or mild up to severe, especially with cardiovascular stress ${ }^{(1)}$.

Different varieties of myocardial pathology are associated with HFPEF, including hypertrophic cardiomyopathy, restrictive cardiomyopathy, and infiltrative cardiomyopathies. However, some HFPEF patients still present without identifiable myocardial pathology ${ }^{(3)}$. Aetiology of hypertension, atrial fibrillation (AF) and coronary artery disease (CAD) is typically common in HFPEF, while the elderly and women are the most affected patients ${ }^{(1,3)}$. Most patients with HFPEF present in stage $\mathrm{C} \mathrm{HF}$, as they have typical HF symptoms with evidence of structural cardiac abnormality including LVH, myocardial scar, aortic sclerosis, mitral annular calcification, or atrial dilation ${ }^{(3)}$. Hospitalization rate is similar to or even higher than that of SHF, while survival rate is still high but better than that reported in $\mathrm{SHF}^{(6)}$.

\section{Epidemiology}

\section{Prevalence}

The prevalence of asymptomatic diastolic dysfunction is approximately $>25 \%$ in the middle-aged healthy population, which is associated with progression into symptomatic HF and poor prognosis ${ }^{(3)}$. Various studies reported a mean prevalence of HFPEF as $56 \%$ (range $40-71 \%$ ) in HF population $^{(1,3,4,6,7)}$. The overall prevalence of HFPEF is estimated to be $1.1-5.5 \%$ of the general population, as it has increased from about one-third to more than $50 \%$ of all HF population over the last two dec$\operatorname{ades}^{(3,7)}$.

Data from HHPEF registries revealed that elderly, females, and hypertensive patients are the most commonly affected groups. The average age of patients is from 73 to 79 years, and women represent $61 \%$ to $76 \%$ of them ${ }^{(1,3)}$. However, age $<70$ years and male gender don't exclude the diagnosis of $\mathrm{HFPEF}^{(3)}$. How to explain this? Physiologically, the elastic properties of the heart and great vessels decrease with aging and eventually lead to systolic hypertension and myocardial stiffness together with reduced ventricular filling which induce diastolic dysfunction. Moreover, co-morbidities like $C A D, A F$ and diabetes mellitus are very common in elderly patients. Women are more susceptible to HFPEF due to sex specific responses to hypertension and diabetes mellitus that increase the cumulative effects of aging on diastolic function $^{(4,7)}$.

\section{Co-Morbidities}

Patients with HFPEF, in comparison with patients with SHF, have a higher likelihood of hypertension with prevalence up to $88 \%$. Other co-morbidities, or better called as "other aetiologies", include CAD (approximately 40\%-50\%), diabetes (approximately $30 \%$ ), atrial fibrillation (approximately 30\%40\%) and obesity (approximately 40\%). Renal impairment, chronic obstructive airway disease (COPD) and anemia are common in

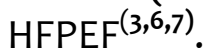

\section{Morbidity}

HFPEF has similar rates of hospital readmissions, HF re-admissions, and NYHA functional class progression, as reported in $\mathrm{SHF}^{(6,7)}$. HF registries have demonstrated that $46-51 \%$ of hospitalized acute HF patients have HFPEF, many of these hospitalizations are re-admissions, with a rehospitalization rate of $29 \%$ within $60-90$ days, and a median time to re-hospitalization of 29 days $^{(6,8)}$. The clinical factors precipitating hospitalization are commonly uncontrolled hypertension and $\mathrm{AF}$, while non-cardiac factors HFPEF include COPD, impaired kidney function and infection ${ }^{(7,8)}$. 


\section{Mortality}

Mortality rate in HFPEF is variable across HFPEF registries and studies, but it is generally associated with high mortality rate. The short-term mortality rate is between 5 and $9.5 \%$ while the long-term ( 5 years) mortality ranges from 52 to $74 \%$. Annual mortality rate ranges from about 3.5 to $6 \%$ in 3 of the large randomized clinical trials ${ }^{(8)}$ to about $15 \%$ in the observational communitybased Framingham Study ${ }^{(7)}$. A recent metaanalysis of 7688 patients with HFPEF followed-up for about 4 years found that presence of $C A D$ is a major risk factor for mortality in HFPEF with estimated overall mortality $32 \%$ and about $8 \%$ annual mortality rate. CAD can negatively affect early and late diastole, which predispose to significant diastolic dysfunction ${ }^{(7)}$.

The Framingham Heart Study showed that HFPEF patients had lower 5-year mortality compared with those with SHF. The annual mortality in patients with HFPEF was $8.7 \%$ compared with $3 \%$ in matched controls and was $18.9 \%$ for SHF compared with $4.1 \%$ in matched controls over 6.2 years ${ }^{(7,8)}$. However, data from the general population of unselected patients reported that mortality of HFPEF may be comparable to that of $\mathrm{SHF}^{(8,9)}$

How can we accept this controversy in mortality? The clue is the criteria of HFPEF diagnosis, is it LVEF $>50 \%,>45 \%$, or $40 \%$ ? This could reflect the variability in the mortality outcome. Furthermore, diversity in demographic, clinical characteristics and presence of co-morbidities could explain this controversy $^{(9)}$. One more explanation is age, when $\mathrm{HF}$ patients were classified as $<65$ years and $\geq 65$ years, mortality in HFPEF was similar to that in patients with SHF when patients were older than 65 years; but among patients younger than 65 years, mortality was lower in those with $\operatorname{HFPEF}^{(3,9)}$.

\section{Etiology}

HFPEF has heterogeneous aetiologies, the only similarity between them is preserved LVEF and evidence of diastolic dysfunction. Several recognized cardiac and non-cardiac disorders are associated with HFPEF. Myocardial disease including restrictive cardiomyopathy, hypertrophic cardiomyopathy, and infiltrative cardiomyopathies. Aortic and mitral valve lesions that affect the intracardiac heamodynamics like significant stenosis or regurge. Pericardial disease, like constriction and tamponade. Extracardiac aetiologies like high output states (e.g. Thyrotoxicosis and arterio-venous fistula). However, some patients who present with HFPEF have no identifiable myocardial pathology ${ }^{(3)}$.

Characteristically, presence of hypertension and LV hypertrophy (LVH) is evident in $88 \%$ of $\operatorname{HFPEF}^{(3,6)}$. Hypertension predisposes to atrial fibrillation (AF) due to high LV filling pressures and increased left atrium (LA) size. Therefore, new-onset AF is common in HFPEF, which reduces LV filling time and lead to loss of the atrial kick. Hypertension and AF precipitate symptomatic HF, pulmonary oedema and consequent hospitalization ${ }^{(3,7,8)}$.

The prevalence of $\mathrm{AF}$ is considerable in both epidemiological studies (30\% to $40 \%$ ) and randomized controlled trials of HFPEF (20\% to $30 \%$ ). AF is associated with worsening of NYHA functional class and quality of life, lower 6 minute walking distance, and larger left atrial diameter than those without $\mathrm{AF}^{(6)}$. In the CHARM (Candesartan in Heart Failure) study, atrial fibrillation was associated with adverse cardiovascular outcomes irrespective of baseline LVEF. High heart rate, loss of atrial systole, irregular cycle length with disabling of the FrankStarling mechanism are the possible mechanisms by which atrial fibrillation confers a worsened clinical status in $\operatorname{HFNEF}^{(10)}$. 
CAD and diabetes are important aetiologies of HFPEF which overlap with hypertension and lead to ventricular long axis dysfunction and impairment of both diastolic and systolic functions, even with preserved $\operatorname{LVEF}^{(7,8)}$. Tissue Doppler Imaging (TDI) can accurately assess ventricular function by detecting small myocardial velocities, namely peak mitral annular systolic and peak early diastolic velocities. Many studies reported that both velocities and the respective excursions are reduced in patients with HFPEF when compared to agematched controls ${ }^{(5)}$.

\section{Pathophysiology}

The pathophysiology of HFPEF of is not fully explored, mainly because of the heterogeneous scope of the affected patients with diverse aetiologies and pathophysiological mechanisms ${ }^{(4,7)}$. The term "preserved" in HFPEF does not really mean "absolutely normal" systolic function, as LVEF gives impression about global LV systolic function which is subject to interobserver and other technical bias. Besides, subtle systolic dysfunction can't be precisely assessed by conventional M-Mode or $2 \mathrm{D}$ echocardiography ${ }^{(11)}$. Long axis ventricular function, assessed by TDI, has shown variable degree of dysfunction in $\operatorname{HFPEF}^{(5,11)}$. Orientation of the myocardial fibres is affected by ageing, hypertension, ischemia, diabetes and LVH. These changes have negative effects on global myocardial architecture and ventricular twist \& recoil during relaxation, which result in reduced ventricular long axis motion and ventricular suction $^{(4,5)}$.

Systole and diastole are closely entangled in the cardiac cycle, so any myocardial pathology, i.e. LVH or fibrosis, will affect systole the way it affects diastole. This explains why both peak annular systolic and early diastolic velocities, assessed by TDI, are equally affected by myocardial fibrosis. The peak early diastolic velocity (a motion of the ventricular base during early diastole), has been proven to be a powerful predictor of prognosis in $\mathrm{HF}$ regardless LVEF value ${ }^{(5,11)}$. Ventricular suction is majorly determined by the strength and coordination of the previous systole which affects early diastolic filling, and vice versa, incoordinate systolic contraction prolongs isovolumic relaxation and further impairs diastolic function ${ }^{(4,5,11)}$.

\section{Molecular and Structural Levels}

Several changes have been observed in HFPEF on the molecular and structural levels within the cardiac myocytes ${ }^{(4)}$. Titin is an elastic molecule inside the sarcomere and is the main determinant of the cardiomyocytes passive stiffness. In patients with HFPEF, there is a change in the expression of titin isoforms with increased expression of the stiffer isoform (N2B) and its degree of phosphorylation over the N2BA, the more compliant isoform. These changes contribute to the increased ventricular stiffness $^{(12)}$.

Increased ventricular and arterial stiffness is accelerated by extracellular matrix and collagen deposition. This stiffness induces stress-induced hypertension and worsens diastolic dysfunction. Moreover, there is a shift in myocardial metabolism from glucose to free fatty acids use because of frequent co-morbidities (e.g. obesity, metabolic syndrome, and type 2 diabetes) ${ }^{(13)}$.

Endomyocardial biopsies in HFPEF reveal significant changes in the cardiomyocytes including: hypertrophied and larger diameters, elevated cardiomyocyte resting tension, greater stiffness and increased density of myofilaments. Grossly, concentric LV remodelling with normal LV volumes is a landmark in $\operatorname{HFPEF}^{(12,13)}$. 
Functional Level (Focus on Diastolic Dysfunction)

The principal abnormality in HFPEF occurs in the diastolic properties. Diastolic dysfunction plays a central role in the pathophysiology of HFPEF, since most patients present with delayed myocardial relaxation and increased ventricular stiffness ${ }^{(1,2,4)}$, this is why HFPEF was often referred to as diastolic HF. Diastolic dysfunction occurs in HFPEF due to changes in the passive properties of the ventricle with increased ventricular stiffness and altered myocardial relaxation $^{(1,3)}$. Delayed myocardial relaxation is caused by reduced calcium kinetics due to reduced activity of SERCA2, the main protein responsible for the reuptake of calcium back to sarcoplasmic reticulum, while increased ventricular stiffness is a sequel of the changes involving the extracellular matrix and the cardiomyocytes ${ }^{(12,13)}$.

LV abnormalities in diastolic dysfunction include abnormal active LV relaxation and stiffness (increased LV passive stiffness). This is associated with a marked increase in LV end-diastolic pressure (LVEDP) and pulmonary venous pressure, which leads to dyspnea during exercise that might progress to pulmonary edema ${ }^{(13)}$. FrankStarling mechanism is disabled in HFPEF as a result of impaired LV filling which can lead to exercise intolerance due to failure of cardiac output increase during exercise $^{(14)}$. Patients with HFPEF are very sensitive to any change in the central volume due to increased ventricular filling pressure, the end product of instantaneous increase of ventricular and arterial stiffness $^{(12)}$.

\section{Extra Cardiac Level}

Many extracardiac abnormalities exist in HFPEF involving mainly the arterial system, which include: increased arterial stiffness, impaired ventricular-arterial coupling, en- dothelial dysfunction, reduced vasodilator reserve, chronotropic incompetence, and abnormal exercise-induced vasodilatation $(13,14)$. Renin-Angiotensin-Aldosterone System (RAAS) is activated leading to increaseed sodium and water retention in the kidneys. Impaired renal function and renal arterial atherosclerosis in the elderly may also contribute to uncontrolled hypertension and excessive fluid retention ${ }^{(14)}$.

\section{Diagnosis of HFPEF}

HFPEF is mainly a diagnosis of exclusion, with heterogeneous aetiologies and differential diagnosis ${ }^{3}$. Clinically, patients may be asymptomatic at rest, but highly symptomatic with mild to moderate exertion. Likewise, functional parameters of diastolic and systolic functions may be normal or slightly abnormal at rest, and they shift to the abnormal side with stress and over time. Therefore, stress test for HFPEF could be very important in diagnosis, however it is still not included in current guidelines ${ }^{(1)}$. Natriuretic Peptides (NPs) are not elevated in all patients with HFPEF, because wall stress (the stimulus of NPs release) may be normal in a non-dilated, hypertrophied ventricle, thus NPs will be released only from the atria, explaining the slightly elevated NT-pro BNP levels in a set of symptomatic HFPEF patients. On the other hand, NPs levels are very high when AF exists, which is another limitation of the its diagnostic value in $\mathrm{HFPEF}^{(13)}$.

\section{Current Diagnostic Criteria of HFPEF}

The current European Society of Cardiology (ESC)/Heart Failure Association (HFA) recommendations $^{(1)}$ require: 1) signs and/or symptoms of heart failure, 2) an ejection fraction above $50 \%$ and a non-dilated ventricle (LV end-diastolic volume $<97 \mathrm{ml} / \mathrm{m}^{2}$ ), as well as either 3) direct evidence of diastolic dysfunction or indirect evidence, like 
elevated natriuretic peptides (NPs). Evidence of diastolic dysfunction can be confirmed by a) invasive measurements; b) tissue Doppler imaging findings; and c) com- bination of elevated natriuretic peptides and echocardiographic indexes of LV diastolic function/LV filling pressures (Figure 1).

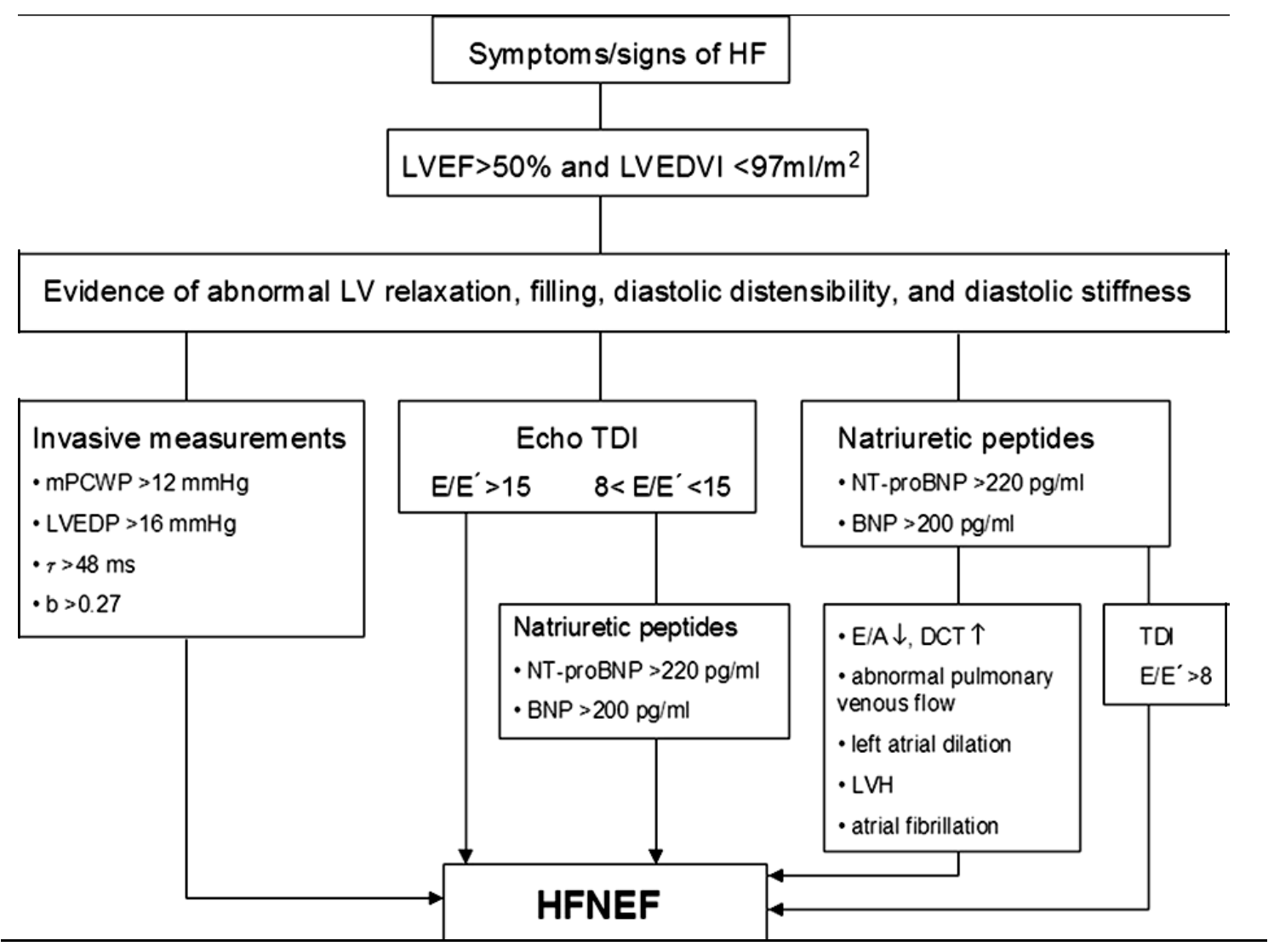

Figure 1: Diagnosis of HFPEF as suggested by the Working Group of the European Society of Cardiology $^{(1)}$. HF= heart failure; $L V=$ left ventricle; $b=$ left ventricular passive stiffness; $D C T=$ deceleration time; $E / A=$ ratio of early to late diastolic peak mitral inflow velocities; $L V E D V I=$ left ventricular enddiastolic volume index; $\mathrm{LVH}=$ left ventricular hypertrophy; $\mathrm{mPCWP}=$ mean pulmonary capillary wedge pressure; $\tau=$ time constant of the isovolemic pressure decline; TDI= tissue Doppler imaging; $\beta=$ stiffness constant; $B N P=B$-type natriuretic peptide; $E / E^{\prime}=$ transmitral peak velocity during early relaxation to early diastolic peak mitral annulus velocity; $L V=$ left ventricular; $L V E D P=$ left ventricular enddiastolic pressure; LVEDV= left ventricular end-diastolic volume; NT-pro BNP= N-terminal pro-B-type natriuretic peptide; HFNEF= heart failure with normal left ventricular ejection fraction.

\section{Echocardiography}

Assessment of LV diastolic function by conventional Doppler ECG using mitral inflow and pulmonary venous flow patterns has been used for many years with proven limitations in clinical practice, as reported in CHARM PRE SERVED study ${ }^{(10)}$. Recently,
TDI has been widely accepted as a sensitive and accurate echocardiographic technique for evaluation of diastolic function (Table 1). TDI derived myocardial velocities continuously decline from normal to advanced LV diastolic dysfunction in which $\mathrm{E}=$ decreases and the $\mathrm{E} / \mathrm{E}=$ ratio continuously increases $^{(5,11)}$. 
Table 1: Commonly Used Echocardiographic Measurements of Diastolic Dysfunction

\begin{tabular}{|c|c|c|c|c|c|}
\hline Criteria & $\begin{array}{l}\text { Normal } \\
\text { adult }\end{array}$ & $\begin{array}{l}\text { Impaired } \\
\text { relaxation } \\
\text { (grade 1, } \\
\text { mild) }\end{array}$ & $\begin{array}{l}\text { Pseudonormal } \\
\text { (grade } 2 \text {, } \\
\text { moderate) }\end{array}$ & $\begin{array}{l}\text { Reversible } \\
\text { restrictive } \\
\text { (grade 3, } \\
\text { severe) }\end{array}$ & $\begin{array}{l}\text { Irreversible } \\
\text { restrictive } \\
\text { (grade } 4 \text {, } \\
\text { severe) }\end{array}$ \\
\hline \multicolumn{6}{|l|}{ Transmitral PW Doppler } \\
\hline - E/A ratio & $1-2$ & $<1$ & $1-1.5$ & $>1.5$ & $1.5-2$ \\
\hline - IVRT (ms) & $70-90$ & $>90$ & $<90$ & $<70$ & $<70$ \\
\hline - DT (ms) & $150-240$ & $>240$ & $150-200$ & $<150$ & $<150$ \\
\hline - Valsalva & Negative & Positive & Positive & Positive & Negative \\
\hline Pulmonary venous flow & & & & & \\
\hline $\begin{array}{l}\text { - S/D ratio } \\
\text { Mitral inflow propagation } \\
\text { velocity }(\mathrm{cm} / \mathrm{s})\end{array}$ & $>1$ & $>1$ & $<1$ & $<1$ & $<1$ \\
\hline $\begin{array}{l}\quad-\mathrm{V}_{\mathrm{p}} \\
\text { Tissue Doppler Imaging }\end{array}$ & $>55$ & $>45$ & $<45$ & $<45$ & $<45$ \\
\hline $\begin{array}{l}\text { - } E^{\prime} \\
\text { LA measurements }\end{array}$ & $>8$ & $<8$ & $<8$ & $<8$ & $<8$ \\
\hline - LAVI & $<34 \mathrm{ml} / \mathrm{m}^{2}$ & $<34 \mathrm{ml} / \mathrm{m}^{2}$ & $>34 \mathrm{ml} / \mathrm{m}^{2}$ & $34 \mathrm{ml} / \mathrm{m}^{2}$ & $>34 \mathrm{ml} / \mathrm{m}^{2}$ \\
\hline
\end{tabular}

$\mathrm{PW}=$ pulse wave; $\mathrm{E} / \mathrm{A}=$ ratio of $\mathrm{E}-$ to $\mathrm{A}$-wave velocities; IVRT= isovolumic relaxation time; $\mathrm{DT}=$ deceleration time; $D=$ peak D-wave velocity; $S=$ peak S-wave velocity; TDI= pulse wave tissue Doppler imaging; $V_{p}=$ propagation velocity; $E^{\prime}=$ early diastolic peak mitral annulus velocity; $L A V I=$ left atrial volume indexed to body surface area. Using TDI, all patients with an $\mathrm{E} / \mathrm{E}^{-}$ratio $>15$ had a mean diastolic LV pressure $>12 \mathrm{~mm} \mathrm{Hg}$ (elevated LV filling pressure). Thus, the diagnostic criteria for HFPEF is presence of $E / E^{=}$ratio $>15$ in a patient with typical symptoms and signs of HF and LVEF $>50 \%$. However, in suspected patients with suspected HFPEF but with an $E / E^{2}$ ratio between 8 and 15 , further measurements and evaluation should confirm or rule out the diagnosis $\operatorname{HFPEF}^{(1)}$.

\section{Natriuretic Peptides}

NT-pro BNP is an established biomarker for the exclusion of suspected HF in patients presenting to the emergency with dyspnea of unknown origin. NT-pro BNP was found to be related to the severity of LV diastolic dysfunction in patients with HFPEF. Therefore, it has been used to distinguish a normal from a "pseudonormal" LV filling pattern $^{(15)}$. HFPEF diagnosis is confirmed when BNP level $>200 \mathrm{pg} / \mathrm{ml}$ or an NT-pro BNP level $>220 \mathrm{pg} / \mathrm{ml}$ in patients with $\mathrm{HF}$ symptoms, LVEF $>50 \%$, and an ambiguous $\mathrm{E} / \mathrm{E}=$ value between 8 and $15^{(1)}$. However, there are some limitations regarding the use of these biomarkers, as it is already high in older age group, $\mathrm{AF}$ and many other $\mathrm{HF}$ comorbidities. Hence, it is recommended that BNP and NT-pro BNP should be mainly used for the exclusion of HFPEF, with upper lim its for exclusion of 100 and $120 \mathrm{pg} / \mathrm{ml}$, respectively ${ }^{(1)}$.

\section{Invasive Evaluation}

HFPEF diagnosis is confirmed when LVEDP or pulmonary capillary wedge pressure are elevated in the presence of HF symptoms and an LVEF $>50 \%$. Constrictive physiology may confound the HFPEF diagnosis, however, TDI can differentiate as $E=$ is low in HFPEF but normal in constriction. Though right heart catheterisation may be useful in selected patients for differential diagnosis of dyspnoea in suspected patients with HFPEF, no diagnostic gold standard exists for $\operatorname{HFPEF}^{(12)}$.

\section{Differential Diagnosis}

Differential diagnosis of HFPEF is broad and diverse, but it should be considered during the initial evaluation of suspected patients (Table 2). Hypertension is the most common cause of HFPEF, then come CAD with transient or stress induced LV dysfunction, 
AF and diabetes mellitus which contribute in the development of $\operatorname{HFPEF}^{(16)}$.

Concentric LV remodelling or hypertrophy is commonly present in patients with HFPEF. The echocardiogram is more sensitive than the electrocardiogram (ECG) for the diagnosis of LVH. In addition to hypertension, LVH may be due to other causes of LV pressure overload, such as aortic stenosis or aortic coarctation. Detection of LVH in the absence of an apparent cause for LV pressure overload supports the diagnosis of hypertrophic cardiomyopathy, which is typically regional (septal or apical), but may be global as well ${ }^{(5,11)}$. Presence of LVH and low voltage ECG with or without pseudoinfarction $Q$ waves raise suspicion for the diagnosis of infiltrative cardiomyopathy. Amyloidosis is the most common infiltrative disorder, which carries a poor prognosis $^{(17)}$ In the absence of hypertrophy, other infiltrative processes include sarcoidosis and Gaucher's disease. Pulmonary sarcoidosis may cause pulmonary hypertension and right-sided $\mathrm{HF}^{(16)}$. Restrictive disorders and pericardial diseases are rare and may be associated with LVH or normal LV mass. Endomyocardial disorders include: endomyocardial fibrosis; the hypereosinophilic syndrome; and carcinoid $^{(16,18)}$. LV volume overload should suspect aortic or mitral regurgitation. Other causes of LV volume overload include: high cardiac output because of cardiac (i.e. ventricular septal defect, or patent ductus arteriosus) or noncardiac causes (i.e. Thyrotoxicosis, arteriovenous shunt, chronic anemia, or chronic liver disease) ${ }^{(5,16)}$. In certain patients with HFPEF of unknown aetiology, it is necessary to differentiate between the diagnosis of pericardial disorders with constrictive physiology and restrictive disorders. The diagnosis of pericardial disease may require cardiac $\mathrm{CT}$, magnetic resonance imaging to identify pericardial thickening or even invasive evaluation (i.e. right heart catheterization) ${ }^{(18)}$.

Table 2: Differential diagnosis in a patient with HFPEF

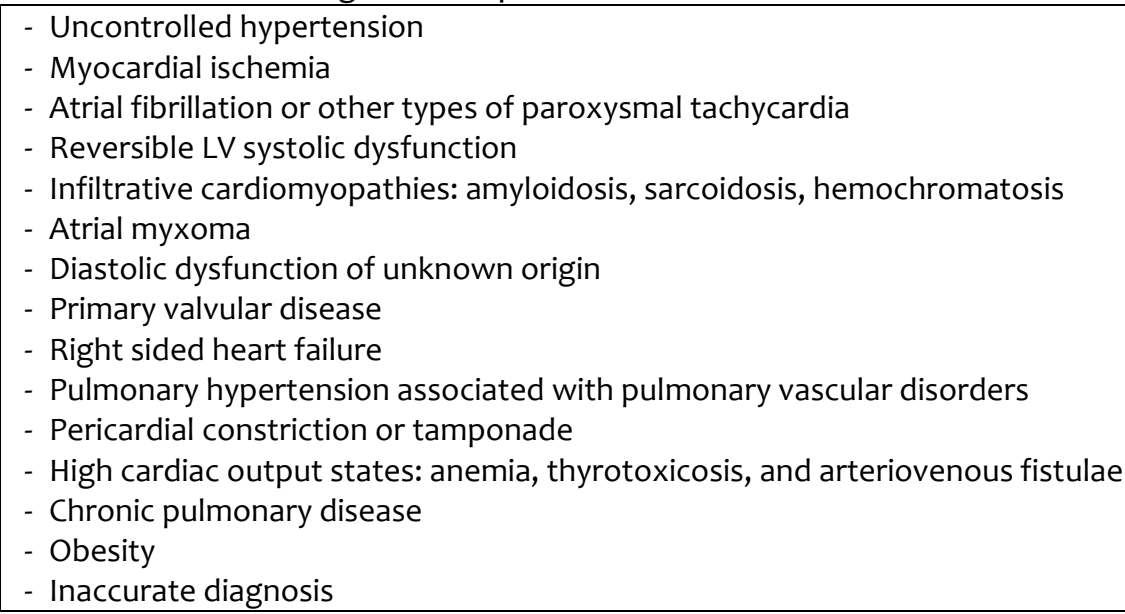

Right ventricular (RV) dysfunction is most commonly secondary to LV dysfunction, in which pulmonary hypertension is predominant. Pulmonary hypertension may exist due to pulmonary thromboembolic disorders and intrinsic lung disease, which lead to RV dysfunction. RV infarction may cause $\mathrm{RV}$ dysfunction and, sometimes, it can cause LV dysfunction resulting from ventricular interaction, high LV diastolic pressure and reduced LV compliance ${ }^{(16)}$.

\section{Treatment}

Therapeutic interventions in HFPEF are still relatively empirical and not evidence based 
despite the clinical and epidemiological significance of HFPEF. Few randomized clinical trials have been conducted in HFPEF patients but did not show survival benefit. Most of therapeutic agents used in HFPEF are recommended based on consensus viewpoint, expert opinion, or data obtained from observational studies ${ }^{(13)}$. Therefore, the prognosis of HFPEF has remained unchanged over time, contrasting with the survival benefit achieved in SHF patients ${ }^{(19)}$.

\section{Targets to Treat}

Management of HFPEF patients includes control of physiological factors: blood pressure, heart rate, blood volume, and myocardial ischemia that are known to affect ventricular relaxation. Proper management of the diseases known to cause HFPEF is essential, as CAD, hypertension, or aortic stenosis ${ }^{(20)}$.

There are important targets to treat and/or prevent HFPEF, which include:

1- Aggressive control of blood pressure, which could prevent and control HFPEF, reduce the number of HF hospitalizations, reduce left ventricular hypertrophy and improve ventriculararterial coupling.

2- Reduction of ventricular filling pressures by use of diuretics and restriction of salt intake.

3- Maintenance of sinus rhythm, which preserves atrial contraction.

4- Control of heart rate and prevention of tachycardia (as tachycardia shortens diastole duration).

5- Treatment and prevention of underlying co-morbidities.

It is logical consensus that appropriate management of hypertension, AF, CAD and diabetes is recommended to prevent $\mathrm{HFPEF}^{(3)}$. Regression of LVH is an important target for prevention of HFPEF, as LVH plays an important role in the development of $\operatorname{HFPEF}^{(4,12,13)}$. In hypertensive patients, reduction of $\mathrm{LVH}$ with antihypertensive treatment is associated with less HF hospitalization. Moreover, data from the Cardiovascular Healthy Study without a history of previous myocardial infarction has shown that LV hypertrophy, per se, is a predictor of $\mathrm{HF}$ progression, independent of different demographic criteria or co-morbidities $^{(21)}$. This is why blood pressure control is principal for treatment of HFPEF (class I, level $A$ ) while other recommendations are with evidence level $C^{(3)}$.

\section{Angiotensin Converting Enzyme (ACE) In- hibitors and Angiotensin Receptor (AT) Blockers}

Presence of high prevalence of hypertension, LV hypertrophy and diabetes in HFPEF has classified the indication of ACE inhibitors or AT blockers as compelling indication. However, the 3 trials evaluating the use of Candesartan (the CHARM-PRESERVED study ${ }^{(22)}$, Irbesartan (the I-PRESERVED study $^{(6)}$ and Perindopril (the PEP-CHF study ${ }^{(23)}$ in HFPEF did not provide survival benefit.

Valsartan use in hypertension patients and LV diastolic dysfunction (the VALIDD study ${ }^{(24)}$ has shown that blood pressure lowering with Valsartan-based regimen versus other regimens resulted in similar reduction in blood pressure and an improvement in diastolic relaxation in both groups $^{(24)}$. However, despite the absence of survival benefit or even morbidity reduction in these trials, ACE inhibitors should be considered in all patients with HFPEF with symptomatic atherosclerotic cardiovascular disease or diabetes and one additional risk factor ${ }^{(3)}$.

\section{Beta Blockers}

Beta-blockers are one of the potential cornerstones in the management of hypertension, so, they should theoretically confer 
great benefits in HFPEF. They have a strong indication in patients with history of prior myocardial infarction and in patients with $\mathrm{AF}$ requiring control of ventricular rate ${ }^{(16)}$. Beta-blockers can reduce the heart rate, increase the duration of diastole and hence ventricular filling time, decrease myocardial oxygen requirements, lower blood pressure and, more importantly, induce regression of LVH. The drawbacks of Beta blockers are reduction of LV contractility and delay of ventricular relaxation ${ }^{(12)}$. Betablockers with vasodilator effect like carvedilol, are expected to have additional benefits as they can reduce arterial stiffness, adding more control to hypertension and more curbing of diastolic dysfunction pathophysiology, by reduction of LVH and arterial stiffness. SENIORS trial showed that Beta-blockers were beneficial in HF patients regardless LVEF value ${ }^{(25)}$ and observational studies have reported that their use in HFPEF may reduce mortality ${ }^{(51)}$. However, the role of Beta-blockers in HFPEF hasn't been well studied yet.

\section{Aldosterone antagonists}

Physiologically, aldosterone retains $\mathrm{Na}$ and water leading to volume overload. Aldosterone acts on the myocardium and vessels, promoting myocyte hypertrophy, fibrosis and collagen deposition, all of which contribute in HFPEF progression via increased myocardial and arterial stiffness ${ }^{(27)}$. Thus, the use of aldosterone antagonists in HFPEF can be theoretically beneficial by reduction of blood volume, and myocardial and arterial stiffness. However, only a small clinical trial demonstrated that spironolactone improved echocardio-graphic parameters of diastolic dysfunction ${ }^{(28)}$.

\section{Calcium Channel blockers}

Calcium channel blockers (CCBs) have a negative inotropic effect and hence is not recommended in SHF. Diltiazem or verapamil could be considered only in patients with HFPEF in atrial fibrillation requiring control of ventricular rate and with contraindication or intolerance to beta-blockers. Symptom-limiting angina and resistant hypertension are another indications for $\mathrm{CCBs}^{(29)}$.

An important effect of these drugs is slowing heart rate, which enhance calcium removal from the myocyte and calcium reuptake in the sarcoplasmic reticulum. This, in turn, lowers end-diastolic pressure and improves passive ventricular filling which, in the long-term, improves exercise capacity ${ }^{(29)}$. CCBs can improve arterioventricular interaction, and reduce arterial stiffness, which could improve exercise performance in patients with $\mathrm{HFPEF}^{(30)}$.

\section{Diuretics}

Low-sodium diet is recommended for all patients with HFPEF. Diuretic treatment is recommended in patients with HFPEF and clinical evidence of volume overload. Treatment may begin with either thiazide or loop diuretics ${ }^{(\mathbf{2 0})}$. Diuretics play a key role in controlling symptoms of volume overload in HFPEF by reducing central congestion $^{(20)}$. Diuretics are less useful in patients without clinical evidence of volume overload, and because of enhanced preload sensitivity in HFPEF, their use may be hazardous in euvolemic patients ${ }^{(16)}$. There is little data regarding diuretic use in HFPEF. In a retrospective analysis of the ALLHAT Trial, the thiazide diuretic chlorthalidone decreased the incidence of HFPEF in hypertensive patients compared to lisinopril, amlodipine, and doxazosin ${ }^{(31)}$. In the Hong Kong Diastolic Heart Failure Study, patients with HFPEF were randomized to diuretics alone or diuretics plus Irbesartan or Ramipril. Quality of life scores improved in all groups, giving a conclusion that diuretics improve symptoms in $\mathrm{HFPEF}^{(32)}$. 


\section{Digitalis}

Digoxin could confer favourable effects on baroreceptor function and reduction in sympathetic activation and neurohormonal stimulation, suggesting a potential role in $\mathrm{HFPEF}^{(4)}$. In an ancillary analysis of $988 \mathrm{pa}$ tients with HFPEF (EF >45\%) enrolled in the Digitalis Investigations Group (DIG) trial, digoxin was found to have no effect on heart failure mortality or hospitalization. Of note, the majority of HFPEF patients in this trial had ischemic aetiology (56\%), and most of hospitalizations were related to unstable angina ${ }^{(33)}$.

\section{Statins}

Statin therapy could be beneficial in HFPEF due to cholesterol reduction and its pleiotropic effects $^{(31)}$. The preliminary data of statin treatment for 6 months in subjects with primary hypercholesterolemia, without coronary heart disease, showed improvement in diastolic function and myocardial contractility ${ }^{(34)}$.

\section{Future treatment targets}

Treatment strategies for HFPEF did not provide survival benefit or reduce morbidity burden over the last 3 decades, unlike SHF, where significant reduction of mortality and morbidity have been achieved with use of different medications. Therefore, there is a great need for evolution of novel treatment strategies targeting $\operatorname{HFPEF}^{(4,31)}$. These novel strategies are expected to suppress the myocardial signal transduction pathways, which account for prominent cardiomyocyte hypertrophy, downregulation of matrix metalloproteinase (MMPs), up-regulation of tissue inhibitors of matrix metalloproteinase (TIMPs), hypophosphorylation of stiff titin isoforms, and substrate shifts from glucose to free fatty acids. Nitric oxide (NO) is one of those emerging therapies, given its beneficial effects on endothelial, vascular and myocardial functions ${ }^{(35)}$.

\section{Conclusion}

HFPEF is a condition associated with shortness of breath, exercise intolerance, and poor quality of life. The principal cardiac abnormality in HFPEF is LV diastolic dysfunction despite a normal LVEF. Other cardiac abnormalities include: concentric LV hypertrophy, increased left atrial size, unrecognized ischemia, paroxysmal atrial fibrillation, altered left atrial function, chronotropic incompetence, vascular stiffness, peripheral factors, and other co-morbidities. The pathophysiology of HFPEF is a combination of increased myocardial and arterial stiffness, ventricular functional and metabolic abnormalities, which result in delayed LV relaxation and raised LV enddiastolic pressure. Symptoms of HFPEF often get worse or become more obvious during exercise.

Thought HFPEF constitutes about 50\% of HF population, it is still a diagnosis of exclusion, and is still undertreated. Therapeutic interventions failed to reduce its mortality and morbidity burden over the last 3 decades, as most of them are not evidencebased. There is a great need to further explore the pathophysiology of HFPEF, which will lead to better understanding and management of this syndrome. Randomizedcontrolled clinical trials are encouraged to involve precisely selected patients and focus on exercise pathophysiology and neurohormonal evaluation in HFPEF. This will establish the optimal therapeutic approach, which is able to improve survival and reduce morbidity burden.

Conflict of Interest: There is no conflict of interest or financial disclosure. 


\section{References}

1. ESC Guidelines for the diagnosis and treatment of acute and chronic heart failure 2008: the Task Force for the Diagnosis and Treatment of Acute and Chronic Heart Failure 2008 of the European Society of Cardiology. Eur Heart J. 2008; 29 (19): 2388-2442.

2. Kessler KM. Diastolic HF. Diagnosis and management. Hosp Pract (Off. Ed.) 1989;24:137-141.

3. Hunt SA, Abraham WT, Chin MH, et al.; American College of Cardiology Foundation; American Heart Association Focused update incorporated into the ACC/AHA 2005 Guidelines for the Diagnosis and Management of Heart Failure in Adults. A Report of the American College of Cardiology Foundation/American Heart Association Task Force on Practice Guidelines Developed in Collaboration With the International Society for Heart and Lung Transplantation. J Am Coll Cardiol. 2009 14;53(15):e1-ego.

4. From $A M$ and Borlaug BA. Heart Failure with Preserved Ejection Fraction: Pathophysiology and Emerging Therapies. Cardiovascular Therapeutics 29 (2011) e6-e21.

5. Hayley BD and Burwash IG. Heart failure with normal left ventricular ejection fraction: role of echocardiography. Curr Opin Cardiol. 2012 Mar;27(2):169-180.

6. Komajda M, Carson PE, Hetzel S, et al. Factors associated with outcome in heart failure with preserved ejection fraction: findings from the Irbesartan in Heart Failure with Preserved Ejection Fraction Study (I-PRESERVE). Circ Heart Fail. 2011 Jan;4 (1):27-35.

7. Lee DS, Gona P, Vasan RS, Larson MB, et al. Relation of disease pathogenesis and risk factors to heart failure with preserved or reduced ejection fraction: insights from the Framingham heart study of the NHLB Institute. Circulation. 2009; 119 (24): 30703077.

8. Fonarow GC, Stough WG, Abraham WT, et al. Characteristics, treatments, and outcomes of patients with preserved systolic function hospitalized for heart failure: a report from the OPTIMIZE-HF Registry. J Am Coll Cardiol 2007;50:768-777.

9. Smith GL, Masoudi FA, Vaccarino V, et al. Outcomes in heart failure patients with preserved ejection fraction: mortality, readmission, and functional decline. J Am Coll Cardiol 2003;41:1510- 1518.

10. Olsson LG, Swedberg K, Ducharme A, et al. Atrial fibrillation and risk of clinical events in chronic heart failure with and without left ventricular systolic dysfunction: results from the Candesartan in Heart failureAssessment of Reduction in Mortality and morbidity (CHARM) program. J Am Coll Cardiol 2006;47:1997-2004.

11. Garcia EH, Perna ER, Farias EF, et al. Reduced systolic performance by tissue Doppler in patients with preserved and abnormal ejection fraction: new insights in chronic heart failure. Int J Cardiol 2006;108:181-188.

12. Fontes-Carvalho R. and Leite-Moreir A. Heart Failure with Preserved Ejection Fraction: Fighting Misconceptions for a New Approach. Arq Bras Cardiol 2011;96(6):504514.

13. Borlaug B. A. and Paulus W. J. Heart failure with preserved ejection fraction: pathophysiology, diagnosis, and treatment. European Heart Journal (2011) 32, 670679.

14. Borlaug BA, Olson TP, Lam CS, et al. Global cardiovascular reserve dysfunction in heart failure with preserved ejection fraction. J Am Coll Cardiol 2010;56:845-854.

15. Januzzi JL Jr., Camargo CA, Anwaruddin S, et al. The N-terminal pro-BNP investigation of dyspnea in the emergency department (PRIDE) study. Am J Cardiol 2005;95:948954.

16. Heart Failure Society of America, Lindenfeld J, Albert NM, Boehmer JP, et al. HFSA Comprehensive Heart Failure Practice Guideline. J Card Fail. 2010 Jun; 16(6):e1-194.

17. Kyle RA, Gertz MA, Greipp PR, et al. Longterm survival (10 years or more) in $30 \mathrm{pa}$ tients with primary amyloidosis. Blood 1999;93:1062-1066. 
18. Karia DH, Xing YQ, Kuvin JT, et al. Recent role of imaging in the diagnosis of pericardial disease. Curr Cardiol Rep 2002;4:33-40.

19. Owan TE, Hodge D, Herges RM, et al. Trends in prevalence and outcome of heart failure with preserved ejection fraction. N Engl J Med. 2006; 355 (3): 251-259.

20.Borlaug BA. Treatment of heart failure with preserved ejection fraction. Curr Treat Options Cardiovasc Med 2009;11:7987.

21. De Simone G, Gottdiener JS, Chinali M, Maurer MS. Left ventricular mass predicts heart failure not related to previous myocardial infarction: the Cardiovascular Health study. Eur Heart J 2008;29:741-747.

22. Yusuf S, Pfeffer MA, Swedberg K, et al. Effects of candesartan in patients with chronic heart failure and preserved leftventricular ejection fraction: the CHARMPreserved trial. Lancet 2003;362: 777-781.

23. Cleland JG, Tendera M, Adamus J, et al. The perindopril in elderly people with chronic heart failure (PEP-CHF) study. Eur Heart J 2006;27:2338-2345.

24. Solomon SD, Janardhanan R, Verma A, et al. Effect of Angiotensin receptor blockade and antihypertensive drugs on diastolic function in patients with hypertension and diastolic function: a randomised trial. Lancet 2007;369:2079-2087.

25. Dobre $D$, van Veldhuisen D, DeJongste $M J$, et al. Prescription of beta-blockers in patients with advanced heart failure and preserved left ventricular ejection fraction: clinical implications and survival. Eur $J$ Heart Fail. 2007; 9 (3): 280-286.

26.van Veldhuisen DJ, Cohen-Solal A, Böhm $M$, et al. SENIORS Investigators. Betablockade with nebivolol in elderly heart failure patients with impaired and preserved left ventricular ejection fraction: data from SENIORS. J Am Coll Cardiol. 2009; 53 (23): 2150-2158.

27. Weber KT. Aldosterone in congestive heart failure. N Engl J Med. 2001; 345 (23): 16891697.

28. Mottram PM, Haluska B, Leano R, et al. Effect of aldosterone antagonism on myocardial dysfunction in hypertensive pa- tients with diastolic heart failure. Circulation. 2004; 110 (5): 558-565.

29. Gaddam KK and Oparil S. Diastolic dysfunction and heart failure with preserved ejection fraction: rationale for RAAS antagonist/CCB combination therapy. J Am Soc Hypertens. 2009 Jan-Feb;3(1):52-68.

30.Bonow RO, Dilsizian V, Rosing DR, et al. Verapamil-induced improvement in left ventricular diastolic filling and increased exercise tolerance in patients with hypertrophic cardiomyopathy: short- and longterm effects. Circulation 1985; 72:853-864.

31. Davis BR, Kostis JB, Simpson LM, et al. Heart failure with preserved and reduced left ventricular ejection fraction in the antihypertensive and lipid-lowering treatment to prevent heart attack trial. Circulation 2008;118:2259-2267.

32. Yip GW, Wang $M$, Wang $T$, et al. The Hong Kong diastolic heart failure study: A randomised controlled trial of diuretics, irbesartan and ramipril on quality of life, exercise capacity, left ventricular global and regional function in heart failure with a normal ejection fraction. Heart 2008; 94:573-580.

33. Ahmed A, Rich MW, Fleg JL, et al. Effects of digoxin on morbidity and mortality in diastolic heart failure: The ancillary digitalis investigation group trial. Circulation 2006; 114:397-403.

34.Talini E, Di Bello V, Bianchi C, et al. Early impairment of left ventricular function in hypercholesterolemia and its reversibility after short term treatment with rosuvastatin: a preliminary echocardiography study. Atherosclerosis 2008;197:346-354.

35.35-Lam CS, Donal E, Kraigher-Krainer E, Vasan RS. Epidemiologyandclinicalcourse ofheart failure with preserved ejection fraction. Eur J Heart Fail. 2011; 13(1):18-28. 